\title{
Juan Dukuen, Habitus y dominación en la antropología de Pierre Bourdieu. Una crítica desde la fenomenología de Maurice Merleau-Ponty, Buenos Aires: Biblos, 2018,
} 337 pp.

\author{
Esteban Andrés García \\ Universidad de Buenos Aires (UBA) - Consejo \\ Nacional de Investigaciones Científicas y Técnicas (CONICET, Argentina) \\ baneste72@gmail.com
}

\section{INTRODUCCIÓN}

Desde su título, la obra aquí presentada se inscribe en el corazón de diversos entrecruzamientos, no solamente el que supone el diálogo teórico entre dos autores -Bourdieu y Merleau-Ponty- sino también el que se traza entre la filosofía y las ciencias humanas, la fenomenología y la antropología, algunos más específicos como los que anudan la epistemología con la filosofía política o la antropología con la sociología, y aun otros mucho más generales como el que existe entre las teorías y las prácticas. Uno de los peligros más frecuentes que enfrentan los estudios de este tipo es el de que, en cada díada, alguno de los términos de la relación haga primar su perspectiva y termine fagocitando al otro -o a su caricatura asimilada y simplificada-. En este sentido, debe destacarse como uno de los más singulares méritos de este trabajo la atención analítica minuciosa dispensada a cada una de las hebras que componen su entramado, lo que resulta en una obra de enorme y singular interés tanto para los especialistas en las ciencias humanas como en la filosofía fenomenológica por igual. Su autor, Juan Dukuen, también autor de Las astucias del poder simbólico (Bs. As.: Ed. Koyatun, 2010), es profesor en la 
Universidad de Buenos Aires e Investigador del Consejo Nacional de Investigaciones Científicas y Técnicas (Argentina), y alcanza en su obra una perfecta complementación entre una sólida formación en el área de la sociología y la antropología - se trata de la reelaboración de una Tesis Doctoral en Ciencias Sociales- y una igualmente rigurosa investigación filosófica — su investigación doctoral fue dirigida por la Dra. Graciela Ralón de Walton, reconocida especialista en los estudios merleaupontianos, quien prologa la obra-.

Bourdieu forjó sus nociones de habitus y de violencia simbólica no sólo en el cruce teórico de la fenomenología y las ciencias humanas, sino que aquello que lo llevó a "reasumir tópicos fenomenológicos [...] para resolver problemas de la antropología y la sociología" ( $p .35$ ) fueron sus investigaciones empíricas en Argelia y el Béarn francés. Dukuen revaloriza, en este sentido, al Bourdieu antropólogo, mostrando que las nociones clave de su teoría de la práctica (ethos, hexis, habitus) "encuentran fundamento empírico en los estudios de etnología cabil y sobre el celibato de los campesinos en el Béarn francés" ( p. 27). Analógicamente podría decirse que para discutir las ideas de Bourdieu, Dukuen replica y renueva la triple dinámica entre fenomenología, teoría social y práctica que generó las ideas que discute: si Dukuen vuelve a invocar herramientas teóricas fenomenológicas para revisar y enriquecer la teoría sociológica, lo hace no sólo apremiado por las tensiones teóricas internas al pensar bourdiano, sino interpelado por otras prácticas, ya no situadas en Francia o Argelia sino esta vez en Argentina, a partir de su participación en investigaciones empíricas -en diversos equipos de trabajo dirigidos por la Dra. Miriam Kriger - que se enfocaron en los discursos y categorizaciones periodísticas acerca de las "villas miseria" y los "pueblos originarios", o la formación de disposiciones políticas en jóvenes escolarizados. En función de la brevedad de esta recensión deberé pasar por alto las diversas secciones relativas a esta práctica sociológica local que recorren la obra y que, como el lector comprobará, van atando firmemente cada tramo del hilo argumentativo que perseguiré al suelo de la experiencia. Al proponerme aquí sólo bosquejar el recorrido argumentativo general de la obra, omitiré también las referencias a la aguda e incesante discusión que Dukuen establece a lo largo de toda su obra con la exégesis bourdiana clásica y contemporánea, dialogando tanto con especialistas del ámbito sociológico como fenomenológico y merleau-pontiano. En este sentido, sólo observaré que, si bien las cuatro partes que estructuran la obra recorren exhaustiva y detalladamente la evolución del pensamiento de Bourdieu a partir de un análisis de sus principales obras y artículos, la 
apuesta del autor no es agregar una nueva introducción general al pensamiento bourdiano a las varias ya existentes. Más bien, por el contrario, su propósito es evitar las lecturas "manualizadas" y simplificadas, relevar los hilos sueltos de su reflexión, "los caminos perdidos o dejados de lado en el recorrido" (p. 112), los desajustes y las tensiones internas que animan el pensar de Bourdieu considerado "verbalmente" como proceso y "en su estado naciente" más que como pensamiento cristalizado.

En esta tónica, la Primera Parte se propone analizar la "Génesis de las nociones de habitus y violencia simbólica" (1958-1964), centrándose en primer término en las investigaciones antropológicas de Bourdieu en Argelia. Fueron ellas las que señalaron su giro hacia la antropología desde su previa formación filosófica, marcada por un interés manifiesto en las primeras obras de Merleau-Ponty y en la filosofía husserliana. A la rigurosa exposición de la fenomenología del tiempo Dukuen dedica dos secciones puntuales: una relativa a los horizontes temporales en las lecciones husserlianas sobre la conciencia del tiempo y otra a las habitualidades en la fenomenología genética. El primero de estos dos análisis resulta de utilidad para comprender de qué modo Bourdieu "antropologiza" las investigaciones husserlianas acerca de la temporalidad -y en particular la distinción husserliana entre "protenciones" y "expectativas" - para elucidar la experiencia subjetiva del tiempo en el marco del ethos tradicional argelino frente al marco capitalista colonial. El segundo análisis acerca de las habitualidades en Husserl contextualiza el análisis de Dukuen acerca de la génesis de la noción bourdiana del habitus, que comienza a esbozarse en sus estudios de 1962 acerca del ethos/hexis corporal en los campesinos del Béarn francés. La particular comprensión bourdiana del habitus remite explícitamente a Mauss pero, como subraya Dukuen, también recoge la refomulación merleau-pontiana de las habitualidades husserlianas, ya no sólo como "posesiones del yo" sino más específicamente de un "cuerpo propio" configurado por capacidades y disposiciones latentes de comportamiento. Como se observa, ya estos análisis iniciales de la génesis de los conceptos vertebrales de la sociología bourdiana señalan en dirección a Merleau-Ponty, cuyo pensamiento cobrará en las dos últimas partes de la obra de Dukuen cada vez mayor protagonismo en la obra. Llamativamente, es por medio de la lectura de Les aventures de la dialectique (1955) de MerleauPonty que Bourdieu conoce el pensamiento weberiano, el cual lo conducirá a sus reflexiones metodológicas acerca de la relación de imbricación entre explicación y comprensión plasmada en la noción de ethos, asociada con la de habitus. Y es 
también en la exigencia merleau-pontiana formulada en Phénoménologie de la perception (1945) de "encontrar para el pensamiento estadístico un fundamento fenomenológico" que Dukuen ve delinearse la posterior preocupación bourdiana por el sentido del dato estadístico.

En la Segunda Parte de la obra Dukuen analiza las "Primeras sistematizaciones de habitus y violencia simbólica" (1964-1970) a partir de los estudios sociológicos bourdianos acerca de la cultura (fotografía, museos) y la educación francesa. Estos estudios llevan a Bourdieu a confirmar el rol clave que las disposiciones y habitus deben tener en los estudios antropológicos y sociológicos, en la medida en que, productos prerreflexivos de la "interiorización de la objetividad", muestran el modo en que la objetividad "se arraiga en y por la experiencia subjetiva" ( p. 101). Dukuen advierte que, surgido de la necesidad de mediar entre los análisis objetivistas y subjetivistas, el habitus no podría reducirse para Bourdieu a un mero canal de "incorporación" de las condiciones objetivas. Sin embargo, La reproducción (1970) parece mostrar al sistema escolar "reproduciendo" la estructura de relaciones de clase a través la violencia simbólica, violencia reconocida como legítima y a la vez desconocida por los dominados en la medida en que arraiga en habitus, modos perseverantes y transferibles de percibir, comportarse, pensar y hablar. Dukuen reconoce aquí la emergencia clara de lo que denominará el problema del "correlato" entre poder simbólico y violencia simbólica, el cual se mostrará fundado a su vez en un segundo correlato entre estructuras objetivas y subjetivas (habitus). Desde este punto, la obra comenzará a esbozar una lectura original de Bourdieu que recurriendo luego a la fenomenología merleau-pontiana- apuntará a sacar a la luz las fisuras de este "doble correlato" que permitan escapar de un fatalismo o un determinismo según el cual la dominación parecería estar garantizada. Dukuen subraya así, por ejemplo, la necesidad de distinguir entre el poder simbólico como instancia y como capacidad, y entre la violencia simbólica como trabajo de incorporación y como efecto que no está nunca garantizado a priori. Desde esta perspectiva más compleja, el autor muestra que en la teoría de los "campos" que opera, por ejemplo, en "Sobre el poder simbólico" (1977) Bourdieu sobreestima el ajuste de las estructuras objetivas y subjetivas restándole "importancia a las prácticas de incorporación de las disposiciones" (p. 146), además de excluir del análisis la dominación de agentes que están "fuera de campo".

La Tercera Parte de la obra muestra el modo en que la "praxeología" o teoría de la práctica bourdiana, formulada particularmente en Esquisse d'une théorie de la 
pratique (1972) y Le sens pratique (1980), encuentra en el habitus el modo de superar los modos “subjetivista” y “objetivista” del conocimiento. Mediante un pormenorizado análisis de algunos capítulos claves de la Phénoménologie de la perception Dukuen comprueba que la crítica bourdiana al intelectualismo y sus desarrollos acerca del habitus como "esquema corporal" y "libertad condicional" se emparentan directamente con las tesis de aquella obra del fenomenólogo que postulaba una intencionalidad corporal simultáneamente centrífuga y centrípeta. Es también a la luz de los conceptos de tal obra que pueden comprenderse, por ejemplo, las distancias críticas de Bourdieu frente a Sartre. Sin embargo, lejos de la mera "incorporación" de temas fenomenológicos, Bourdieu "retoma el tema fenomenológico de la temporalidad y la formación de habitus corporales reelaborándolo en una teoría de la dominación [...] no desarrollada previamente en Husserl, Schutz o Merleau-Ponty" (p. 162). El autor releva con detalle otra de las fuentes de inspiración de la teoría bourdiana de la práctica, la proveniente de la concepción de Pascal de la creencia. Esta concepción supone "una lectura de las prácticas mundanas como mayormente prerreflexivas, lo cual permite ubicarlo como antecedente de la fenomenología merleau-pontiana" (p. 177). Pero aquello que permitirá a Dukuen realizar una lectura crítica y renovadora del problema bourdiano del "doble correlato" -crítica a la vez "escrita con Bourdieu y contra Bourdieu" (p. 213) - será especialmente la concepción no mecanicista del hábito de Merleau-Ponty, y su análisis de la compleja dinámica de imbricación y desfasaje entre el "cuerpo habitual" y el "cuerpo actual". Esta dinámica introduce la ambigüedad y la indeterminación en el seno de las prácticas y permite abordar el mundo social desde una dialéctica de lo instituido y lo instituyente, así como de la sedimentación, la reactivación y la transformación del sentido: "no hay ajuste entre las prácticas, las condiciones objetivas y las situaciones, sino diferentes grados de complicidad ontológica entre un agente estructurado por un habitus y una situación" ( p. 229). Sólo desde este renovado marco, señala el autor, podrán comprenderse los "habitus desgarrados" ya observados por Bourdieu en sus primeras investigaciones antropológicas, así como la noción de “contradiestramiento" (contre-dressage) propia de las posteriores Méditations pascaliennes.

En la Cuarta Parte, titulada "Aventuras de una teoría de la violencia simbólica", Dukuen confirma, en primer lugar, las aporías a las que conduce el "doble correlato" en los análisis bourdianos concretos del "gusto de necesidad" (La distinction, 1979) y las relaciones de dominación sexual (La domination masculine, 1998). Por ejemplo, en este último trabajo se observa problemáticamente que siendo la violencia 
simbólica -fundamentada en el carácter corporal-disposicional de las prácticaspresa del "doble correlato", Bourdieu no acierta a brindar "ningún fundamento teórico a la posibilidad crítica de las luchas contra la dominación masculina que el libro busca alentar" (p. 282). En ciertas secciones de Le sens pratique (1980), La noblesse d' État (1989) y Méditations pascaliennes (1997) el autor halla, en cambio, elementos que contribuyen a su reelaboración crítica de los rasgos fatalistas de la teoría bourdiana de la dominación. Si desde los primeros estudios antropológicos Bourdieu daba lugar a "habitus escindidos" o "desgarrados" y "ethos contradictorios" o "ambiguos", algunas de sus últimas obras permiten considerar la indeterminación de los efectos del trabajo de violencia simbólica. Pueden advertirse así diversas brechas en distintos niveles del "doble correlato" que Dukuen clasifica con minucia analítica, aquellas presentes en el proceso de formación, reproducción y transformación de los habitus, y que requieren reconocer "grados de dominación" y "márgenes de libertad". Como señala acertadamente el autor, un reconocimiento de este tipo no significa "negar los condicionamientos sociales, sino más bien comprenderlos en su lógica específica, la de la práctica", y esta lógica permite ver en raras y felices ocasiones que "las relaciones de dominación que las prácticas sociales han instituido pueden ser transformadas por prácticas colectivas instituyentes" (pp. $321,322)$. 